\title{
ANÁLISE DE FATORES SÓCIO-ECONÔMICO-CULTURAIS E AMBIENTAIS RELACIONADOS COM O DÉFICT PONDERAL DE CRIANÇAS AO NASCIMENTO EM 1999, EM MANAUS-AM, BRASIL
}

\author{
Fernando Hélio ALENCAR¹, Marcelo Oliveira da FROTA²
}

RESUMO: Investigou-se as causas do nascimento de crianças com défict ponderal, enfatizandose como determinante do Baixo Peso ao Nascer (BPN), o sinergismo entre algumas variáveis sociais, econômicas, culturais e ambientais. A populaçāo estudada compreendeu 371 māes, com seus respectivos recém- nascidos vivos não gemelares, cujos partos ocorreram no período de setembro de 1998 a maio de 1999, em uma maternidade pública de Manaus. Constatou-se a predominância de Recém- Nascidos de Peso Adequado (69,5\%), sendo que 8,9\% e 21,6\%, das crianças se enquadraram nas categorias de Baixo Peso e Peso Insuficiente, respectivamente. Como principais fatores maternos foram detectados a baixa idade, baixa estatura e baixa escolaridade. A análise dos resultados obtidos permite concluir que é elevada a ocorrência de déficit ponderal ao nascer em Manaus, sendo seus principais determinantes $(p<0,05)$ : gravidez na adolescência, início de realizaçāo do pré-natal e ganho de peso durante a gestaçāo. Merecem destaque também o comportamento das variáveis: baixa escolaridade materna, ausência paterna, precariedade na assistência materno- infantil, possivelmente em decorrência de um prénatal mal conduzido, baixo poder aquisitivo das famílias, agravado pelo número excessivo de seus integrantes, o que determina a diluição do poder de compra de alimentos intrafamiliar.

PALAVRAS-CHAVE: peso ao nascer, determinantes, assistência materno-infantil

\section{EVALUATION OF SOCIAL, ECONOMICAL, CULTURAL AND ENVIRONMENTAL AS DETERMINING FACTORS OF WEIGHT DEFICIT OF CHILDREN AT BIRTH IN 1999 AT MANAUS, AMAZONAS STATE, BRAZIL}

ABSTRACT: The present study has investigated the causes of weight deficit at birth, emphasizing the interaction between social, economical, cultural and environmental variables, as the Low Weighted Birth (LWB) determining factors. The population studied comprised 371 moth ers and their live newborn non-twinned children, whose births had occurred in a Manaus public maternity hospital from September 1998 to May 1999. The predominance of Ad equately Weighing of Recently Born Children was $69,5 \%$, whereas $8,9 \%$ and $21,6 \%$ of the

\footnotetext{
${ }^{1}$ Instituto Nacional de Pesquisas da Anazônia - Coordanação de Pesquisas en ciências da Saíne: INPA/CFCS - Rv. Anzié Araújo 2936, Petrópolis, Maraus-Am: GP 69083-000 (falencara.impa.gov br)

${ }^{2}$ Bolsista do programa do Gaeno Federal de Iricialização Cientifica (RIBIC/INEA-AEq:1999)
} 
children were in the low and not enough weight categories, respectively. Young age, short height and low educational status were recorded as the mothers' main characteristics. The analysis of the results allowed us to conclude that the occurrence of weight deficit of children at birth is high in Manaus, and that mothers' low level of education, teenage pregnancy, fathers' absence, and precarious mother-child welfare care, probably a result from badly managed pre-natal welfare planing, are amongst the most important determining factors. The family's low income was also worsened by the occurrence of high numbers of members, which reduces the intra-familiar food purchasing power.

KEYWORDS: birth weight, determining factors, mother-child welfare care.

\section{INTRODUÇÃO}

Há evidências na literatura de que o estado nutricional materno durante a gestaçāo é o principal determinante do peso ao nascer da criança, sendo esta condição biológica, o fator determinante da sua saúde e sobrevivência. Segundo Vitalle et al, 1997; as influências biológicas, sociais, econômicas e nutricionais, vivenciadas antes da gravidez, se refletem na gestação, podendo interagir com fatores obstétricos e comprometer o produto final da gestação. É notório na literatura nacional a carência de informação sobre o estado de saúde do homem no contexto Amazônico. O diagnóstico das condições de saúde, nutrição e sobrevivência dos seus diferentes grupos populacionais, são relativamente escassos, fragmentados, alguns dos quais realizados há mais de duas décadas (Shrimpton et al, 1983; Yuyama et al, 1989; Ferraroni et al, 1991). Especificamente para o Estado do Amazonas é delineado um perfil pluricarencial, caracterizado pela deficiência de micro e macro nutrientes, processos infecciosos, alta prevalência de parasitose gastroentestinal, cárie dental e elevado índice de déficit ponderal em pré- escolares da área urbana e rural. (Giugliano et al, 1981, 1984; Shrimpton et al. 1983; Nagahama et al.,1990). Entretanto, estudo com representatividade populacional, investigando a ocorrência de baixo peso ao nascer, suas causas e conseqüências foi realizado em Manaus por Giugliano et al (1976); há mais de duas décadas. A implicação epidemiológica da ausência destas informações, pode ser avaliada pelo fato de que, crianças com déficit ponderal ao nascer são mais vulneráveis, correndo maior risco de morrer mais prematuramente do que aquelas que nascem com peso normal. Caso sobrevivam, terão episódios de doenças mais freqüentes e demorados, comprometendo o seu desenvolvimento mental, cognitivo e predispondo a criança a complicaçôes futuras, tais como: diabetes, doenças cardíacas e outras condições crônicas (UNICEF 1994,1998).

Neste sentido, o presente trabalho objetivou investigar em uma Maternidade Pública de Manaus, as causas do nascimento de crianças com déficit ponderal, enfatizando-se como fator determinante deste evento, o sinergismo entre as variáveis sociais, econômicas, culturais e ambientais.

\section{MATERIAL E MÉTODOS}

\section{Amostra}

A população estudada envolveu 371 mães, com seus respectivos recém- nascidos 
vivos, não gemelares, cujos partos ocorreram no período de setembro de 1998 a maio de 1999 , na Maternidade Balbina Mestrinho (Manaus Am), a qual atende a população de baixo poder aquisitivo da área metropolitana e rural do Estado do Amazonas.

\section{Coleta de Dados}

A coleta de dados foi obtida apoiando- se em dois fluxos de informaçōes: Primário - por entrevista materna (aleatória) precedente ao parto, utilizando- se questionários padronizados, e aprovados em pesquisas similares, realizadas anteriormente pelo grupo da Nutrição da Coordenação de Pesquisa em Ciências da Saúde - CPCS/INPA. Inqueriu- se as mães a respeito dos fatores sociais, econômicos culturais e ambientais, investigado-se o seu poder de influência na determinação do estado nutricional da mãe e do filho.

Secundário - a partir dos prontuários médicos, obteveram- se informaçōes sobre as condições de saúde e nutrição da mãe e do recém- nascido.

\section{DEFINIÇÃO/PROCEDIMENTOS}

\section{Dos eventos biológicos}

Na avaliação do estado nutricional do recém- nascido, adotou-se a classificação de peso recomendada pela OMS (1980), que estabelece os seguintes parâmetros:

(1) Recém- Nascidos de Baixo Peso (RNBP) - crianças cujo peso ao nascer é igual ou inferior a $2500 \mathrm{~g}$.

(2) Recém- Nascidos de Peso Insuficiente (RNPI) - crianças cujo peso ao nascer fica compreendido entre 2501 e $3000 \mathrm{~g}$.

(3) Recêm- Nascidos de Peso Adequado (RNPA) - crianças com peso ao nascer acima de $3000 \mathrm{~g}$.

\section{Das variáveis estudadas}

Estado Civil - Foi classificado em solteira, casada, viúva, separada e amasiada.

Renda familiar e/ ou do responsável produto da renda auferida por todos os integrantes da família e/ou do seu responsável, expressa em salários mínimos per capita.

Número de pessoas que coabitam com a mãe - variável essencial como agente diluidor da renda familiar.

Peso materno no início da gravidez expresso em quilogramas e de acordo com o registro do cartāo de acompanhamento do prénatal e/ ou via informação do prontuário da mãe.

Peso materno ao final da gravidez este dado foi obtido pesando-se a parturiente em balança Filizola tipo plataforma com precisão de $100 \mathrm{~g}$ e capacidade de $150 \mathrm{Kg}$, estando a parturiente descalça e com vestimenta leve. Após cada pesagem realizouse calibragem da balança em ponto zero, segundo a técnica descrita por Jelliffe (1968).

Aumento de peso durante a gravidez - correspondente à diferença entre o peso final e o peso no começo da gravidez.

Altura da mãe - foi obtida em balança Filizola, acoplada com haste vertical graduada em $0,5 \mathrm{~cm}$, estando a parturiente de pé, descalça, de pés juntos na plataforma da balança com calcanhares e costas o mais próximo na barra de escala de graduação, a cabeça em plano horizontal, em posição reta e olhando a frente, segundo a técnica recomendada por Jelliffe (1968).

Hábito de fumar durante a gravidez - classificou- se as mães como fumantes ou nāo fumantes.

Realização do pré-natal - investigou se, por meio do Cartão do Pré- natal, o período, início e o número de consultas.

Educação da mãe - foi expressa em anos de escolaridade e/ ou de acordo com a 
classificação dos níveis de escolaridade estabelecidos pelo Ministério da Educação.

\section{ANÁLISE ESTATÍSTICA}

Para análise estatística foram utilizados os testes de comparação de duas proporções e quando necessário, o qui- quadrado de Pearson, adotando- se o nível de significância de 0,05, obtidos pelo programa Epi Info 6.0. A associação entre cada uma das variáveis maternas e o peso do recém- nascido foi testada utilizando- se o teste estatístico do $\mathrm{X}^{2}$.

\section{RESULTADOS E DISCUSSÃO}

No presente estudo foram registradas ocorrências de $8,9 \%$ e $21,6 \%$, de crianças com baixo peso e peso insuficiente, respectivamente. $\mathrm{O}$ significado epidemiológico destes resultados é que eles refletem a qualidade de vida ambiental e expectativa de esperança de vida da criança ao nascer em Manaus, estando portanto, o maior percentual destas crianças necessitando urgentemente de melhor alimentação, cuidados e atenção médica. Estes resultados não divergem muito daqueles registrados no Estado do Amazonas por Giugliano et al (1976) e Guedes (1989). Entretanto, superam os percentuais referidos para os países em desenvolvimento (18\%) (Monteiro, 1985). O Unicef (1998), refere que atualmente, nascem, por ano, cerca de 24 milhões de crianças com baixo peso, o que representa cerca de $17 \%$ de todas as crianças que nascem com vida, principalmente, nos países em desenvolvimento. O estudo evidenciou ainda como principais características maternas: baixa idade $(40,4 \%$ com menos de 20 anos de idade), baixa estatura $(88,7 \%$ com altura igual ou inferior a
$1,60 \mathrm{~m})$. Há referência na literatura demonstrando maior predisposiçāo das mulheres, excessivamente jovens e de baixa estatura, a terem conceptos de baixo peso e maior risco de morbi- mortalidade: toxemia, hemorragias, anemias, infecções, complicações no trabalho de parto e mortalidade perinatal. (Fisberg et al.,1997; Vitalle et al.,1997). No presente estudo, o grupo de adolescentes com menos de 15 anos, apresentou o maior percentual de recém- nascidos de baixo peso $(21,7 \%)$; entretanto, a variável altura materna não foi suficientemente sensível para discriminar, nutricionalmente, as crianças pelo seu peso ao nascer. Como determinantes do perfil sócio-econômico da população estudada, constatou- se que $22,1 \%$ das entrevistadas eram māes solteiras ou separadas, $71,1 \%$ referiam baixa escolaridade (analfabetas ou com o primeiro grau incompleto), estando, portanto, totalmente despreparadas para arcarem com a difícil tarefa de suprir as necessidades da família. Cerca de 43,1\% das famílias auferiam uma renda inferior a um salário mínimo e com o agravante de que $92,8 \%$ das mães constituíam famílias numerosas $( \pm 5$ pessoas por núcleo familiar) o que obviamente, determina diluição da renda e restrição do poder de compra de alimentos. Invariavelmente, a pobreza no nível familiar constitui uma das principais causas da desnutriçāo infantil, além de outros fatores de origem social, política, econômica e cultural. Nossos resultados evidenciam que famílias numerosas, de baixa renda e mães analfabetas e/ou com baixa escolaridade apresentaram os percentuais mais elevados de RNBP. No que se refere à assistência médico-social materna, constatou-se que $92,7 \%$ das parturientes realizaram o pré-natal 55,0\% iniciando-o logo no primeiro trimestre da gravidez $(\mathrm{p}<0,05)$; $77,1 \%$ referindo mais de três consultas durante 
o pré-natal, constatando- se ainda, em 45,5\% das parturientes, um incremento ponderal superior a $10 \mathrm{Kg}(\mathrm{p}<0,05)$ no final da gestação. Fisberg et al (1997), relatam ocorrência de RNBP mais freqüente em mulheres que não realizam o pré-natal, ou que o fazem tardiamente e que iniciam a gravidez com peso inferior a $55 \mathrm{Kg}$, ou ainda, tendo um incremento ponderal inferior a $8 \mathrm{Kg}$ no final da gravidez. Segundo estudo realizado pelo Departamento de Agricultura dos Estados Unidos (1990), in: (UNICEF, 1998) os investimentos em cuidados pré-natais às gestantes resultaram em uma relação custobenefício altamente positiva, traduzida em reduçāo dos custos de atendimento médico às crianças durante os dois primeiros meses após o nascimento. No presente estudo, apesar da homogeneidade do grupo estudado, no que se refere à realização do pré-natal, foi expressivo o incremento do baixo peso ao nascer em função do período em que as mães iniciaram o pré-natal e do ganho de peso que as mesmas apresentaram durante a gravidez. $O$ conjunto destas informações deixa evidente que a população estudada enfrenta sérios problemas de inadequação nas condiçôes de saúde, nutriçāo e sobrevivência, tornando-se imperioso o convencimento das māes, quanto à necessidade de realização do pré-natal desde o início da gravidez, tornando compreensível o porque da importância do acompanhamento da sua evolução ponderal, da tomada de sua pressão arterial, da realização dos exames quando solicitados, da educação antitabagismo, da ingestão de bebidas alcoólicas, do comportamento sexual durante a gravidez, da vacinaçāo antitetânica, notadamente, daquelas oriundas da área rural, ou que pretendam ser assistidas em casa e principalmente, na divulgação da importância do aleitamento materno. Deve ser divulgada a idéia de que o nível de escolaridade possibilitará à mãe um melhor gerenciamento das suas tarefas domésticas, dos parcos recursos familiares, dos cuidados com a saúde e nutriçâo da família. Alertar as mães para a importância do acompanhamento mensal do desenvolvimento da criança (peso e altura), viabilizará o diagnóstico precoce da desnutrição, seu tratamento e indiretamente, a prevenção de processos infecciosos. Transferir para as māes, com linguagem acessível, informações precisas de qual deve ser o seu comportamento na vigência da diarréia do filho, no que se refere à higiene, contatos dos contaminantes ou manipuladores de alimentos, utilização do soro de reidrataçāo oral.

\section{BIBLIOGRAFIA CITADA}

Ferraroni,M.J.R; Marinho, H.A; Nagahama, D; Rocha, Y.R; Silva, N.B; Castro, J.S. 1991. Prevalência de enteropatias na cidade de Manaus. Revista de Pediatria 67(1/2).

Fisberg, M; Anti, S.M.A, Yamashiro. S.N. 1997. Baixo peso ao nascimento. IEpidemiologia. Pediatria moderna. 33(1/4): 41-49.

Giugliano, R; Souza, J.U; Shrimpon, R; Giugliano, L.G.1976. Levantamento da freqüência de baixo peso de nascimento em Manaus. Acta Amazonica (1):109-116.

Giugliano, R.; Giugliano, L.: Shrimpton, R. 1981. Estudos nutricionais das populações rurais da Amazônia. I. Várzea do Rio Solimōes. Acta Amazonica, 11(4):773-788.

Giugliano, R.; Shrimpton, R.; Marinho, H. A.; Giugliano,L. 1984.Estudos nutricionais das populações rurais da Amazônia. II. Rio Negro. Acta Amazonica, 14(3-4):427-449.0

Guedes, M.R.1989. contribuição ao conhecimento do estado nutricional e renda 
familiar de gestantes sadias, em relação com seus recém-nascidos. Dissertação de Mestrado, Instituto Nacional de Pesquisas da Amazônia. Fundação Universidade do Amazonas. Manaus, Amazonas, 106p.

Jelliffe, D.B.1968.Evaluación del estado de nutrición de la comunidad; com especial referencia a las encuestas en las regiones en desarrollo. Genebra: Organización Mundial de la Salud. 291p.

Monteiro, C. A .1985. Avaliação do impacto da suplementação alimentar a gestante no controle de baixo peso ao nascer no município de São Paulo.S.P.,Brasil. Revista Saúde Pública, 19:458-474.

Nagahama, D.;Marinho, H. A.; Rocha, Y.; Ferraroni, M.; Silva, N. Castro, J.; Onety, J.A. 1990. Avaliaçāo nutricional e alimentar de pré-escolares de uma creche de Manaus e a influência da entidade no estado nutricional de sua população. Acta Amazonica, 20(único):119-129.

World Health Organization - WHO. 1980. The incidence of low birthweight: a critical review of avaliable information. World Health Statistics Quaterly.

Shrimpton, R. 1973. Food consuption and dietary adequacy accord to income in 1200 families. Manaus, Amazonas, Brasil. Arquivos Latinoamericanos de Nutrición. XXXIV:(4).
Shrimpton, R \& Giugliano, R.1979. Consumo de alimentaçāo e alguns nutrientes em Manaus, Amazonas. Acta Amazonica 9(1): 117-141.

Shrimpton, R; França, T,S; Rocha, Y.R; Golden, M.H.N. 1983. Estudo sobre o estado nutricional em relação ao zinco na Amazônia. Acta Amazonica, 13 (1):73-94.

Fundo das Nações Unidas para a Infância UNICEF, 1994. Situação Mundial da Infância.

Fundo das Nações Unidas para a Infância UNICEF,1998. Situação Mundial da Infância.

Vitalle, M.S.S.; Brasil, A.L.D; De Nobrega, F.J. 1997. Recém-nascidos de mães adolescentes de baixo nível socioeconômico. Revista Paulista de Pediatria, 15 (1):17.

Yuyama, L.K.O; Nagahama, D; Marinho, H.A; Vannucchi, H. 1989. Alimentação e estado nutricional de māes em diferentes estados fisiológicos de um bairro pobre de Manaus. Alimentos e Nutrição., São Paulo, (1):1321.

Submetido à publicação: 27/02/2002.

Aceito: 12/09/2002. 
Tabela 1. Relação entre peso dos recém-nascidos e as variáveis maternas investigadas. Manaus-Am, 1999.

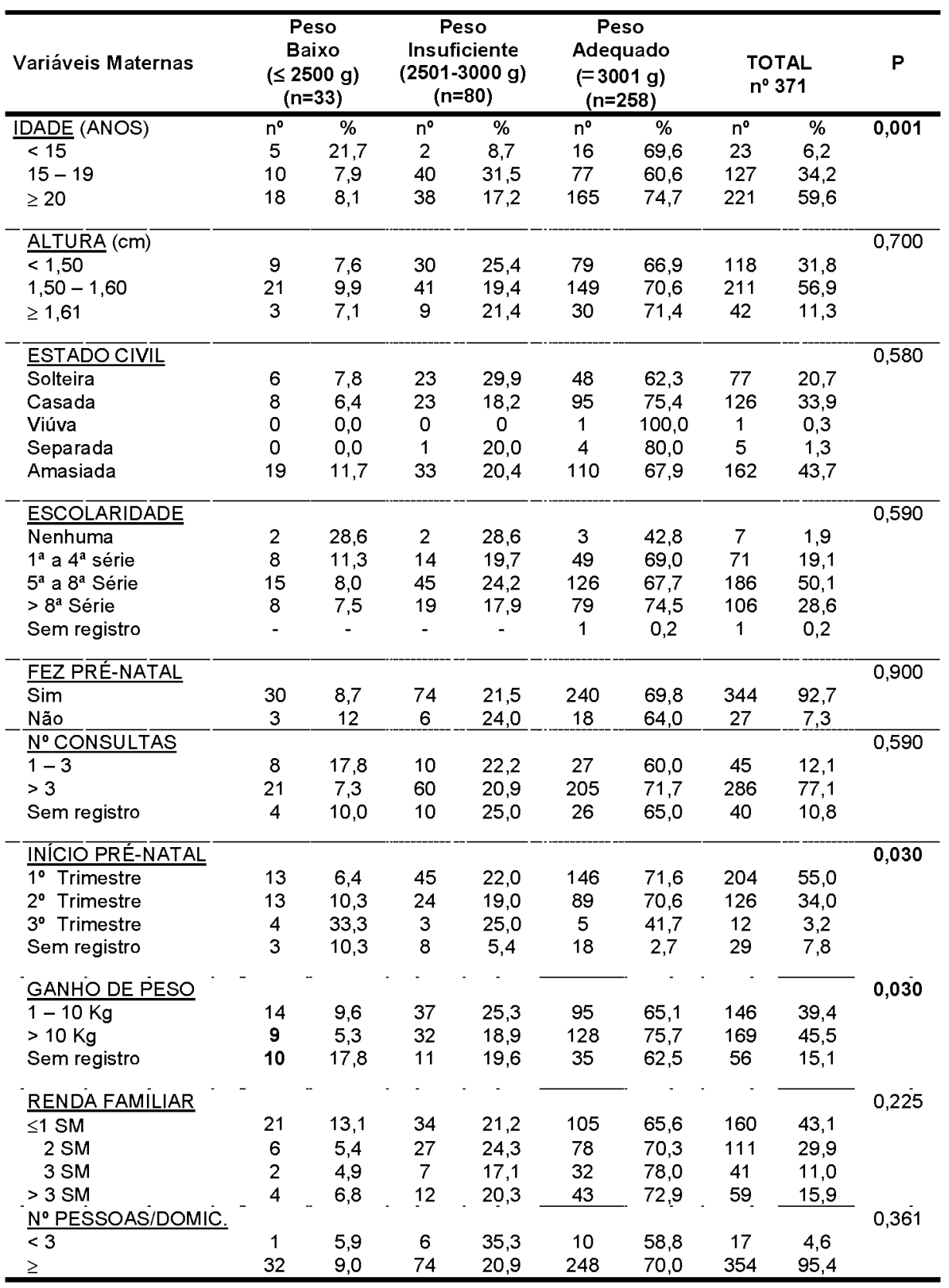

